\title{
DIFFRACTION BY A HYPERBOLIC CYLINDER
}

\author{
BY CLIFFORD O. BLOOM ${ }^{1}$
}

Communicated by J. B. Diaz, January 15, 1968

We investigate the asymptotic behavior as $K \rightarrow \infty$ of the solution $G\left(x, y ; x_{0}, y_{0}: K\right)$ of the following scattering problem $P$ :

(i) $\left[\Delta+K^{2}\right] U=\delta\left(x-x_{0}, y-y_{0}\right), \quad(x, y),\left(x_{0}, y_{0}\right) \in D$;

(ii) $\partial_{n} U=0, \quad(x, y) \in C$;

(iii) $\lim _{p \rightarrow \infty} \int_{\Sigma(\theta, 0) \cap \bar{D}}|\partial U / \partial r-i K U|^{2} d S=0$.

Here $C$ is the left branch of the coordinate hyperbola $(x / h \cdot \cos n)^{2}$ $-(y / h \cdot \sin \mathfrak{n})^{2}=1, \pi / 2<\mathfrak{n}<\pi$. In parametric form $C$ is given by the equations $x=h \cdot \cos n \cdot \cosh \xi, y= \pm h \cdot \sin n \cdot \sinh \xi, \xi \geqq 0$.

$D$ is the infinite two dimensional region bounded by the convex side of $C ; D$ consists of all points $(x, y)$ with elliptic coordinates $(\xi, \eta)$ such

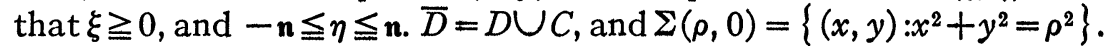

$\Delta$ is the two dimensional Laplacian, $\delta\left(x-x_{0}, y-y_{0}\right)$ is Dirac's $\delta$-function, and $\partial_{n}$ denotes differentiation in the direction of the outward normal to $C$.

Our result is a rigorous asymptotic expansion of the Green's function $G$ as $K(>0) \rightarrow \infty$ that holds uniformly in every closed bounded subset $S_{<}\left(x_{0}, y_{0}\right)$ of the "shadow" $S\left(x_{0}, y_{0}\right)$ of $C . S\left(x_{0}, y_{0}\right)$ consists of those points in $D \cup C$ that cannot be joined to $\left(x_{0}, y_{0}\right)$ by a line segment lying entirely in $D \cup C$.

The asymptotic expansion we get for $G$ confirms the geometrical theory of diffraction by convex cylinders of infinite cross section (see [1]).

Furthermore, our rigorous asymptotic solution of the problem $P$ can be used with certain bounds to obtain asymptotic solutions of a general class of scattering problems with smooth convex boundaries $C^{\prime}$ that coincide with $C$ in neighborhoods of the points of "diffraction"; the points where the boundary of $S\left(x_{0}, y_{0}\right)$ intersects $C$. For example if $C^{\prime}$ is formed by joining a convex $\operatorname{arc} A$ to the "illuminated" part of $C$, then an asymptotic expansion of the solution $G^{\prime}$ in the shadow $S^{\prime}\left(x_{0}, y_{0}\right) \quad\left(=S\left(x_{0}, y_{0}\right)\right)$ can be obtained once it is known, for some positive $N$, that $G^{\prime}\left(x, y ; x_{0}, y_{0}: K\right)=O\left(K^{N}\right)$ as $K \rightarrow \infty$, uniformly in $(x, y),(x, y) \in A$.

${ }_{1}^{1}$ The research described here was done at the Courant Institute of Mathematical Sciences in 1964 under the guidance of Professor J. B. Keller. It was supported by the U.S. Air Force Office of Scientific Research under grant No. AFOSR-537-64. Reproduction in whole or part is permitted for any purpose of the United States Government. 
The following is an outline of the argument we use to obtain an asymptotic solution of the problem $P$. The details of the argument are given in [2].

We first express the problem in elliptic coordinates, and then construct a Fourier expansion of $G$ with respect to the eigenfunctions $P^{(j)}\left(\eta ; \tau_{n}^{(j)}, K\right), j=1,2, n=1,2,3, \cdots$ of the operator $L_{\eta}=d^{2} / d \eta^{2}$ $-(K h \cdot \cos \eta)^{2} . P^{(1)}(\eta ; \tau, K)$ and $P^{(2)}(\eta ; \tau, K)$ are the first and second principal solutions, relative to $\eta=0$, of Mathieu's equation $\left(L_{\eta}+\tau^{2}\right) U=0$ on the interval $-n \leqq \eta \leqq n$. The eigenvalues $\tau_{n}^{(j)}$ are the positive zeros of the entire function $d P^{(j)}(\mathbf{n} ; \tau, K) / d \eta$.

Next we sum the Fourier series to a contour integral $\int_{C} I(\xi, \eta$; $\left.\xi_{0}, \eta_{0}: Z, K\right) d Z / 2 \pi i$, where the integrand $I$ is a meromorphic function of $Z$, and $C$ is a horizontal line in the upper half of the $Z$-plane.

For sufficiently large $K$ the poles of $I$ in the upper half of the $Z$-plane are the zeros $Z_{n}(K)$ of the entire function $F(\mathbf{n} ; Z, K)$ defined as follows. Let $h^{(1)}(\xi ; Z, K)$ be an entire function of $Z$ that satisfies the associated Mathieu equation $d^{2} U / d \xi^{2}+\left[(K h \cdot \cosh \xi)^{2}-(K Z)^{2}\right] U=0$ on $\xi \geqq 0$, and that is asymptotic to $\exp [i K h \cdot \sinh \xi] /(h \cdot \cosh \xi)^{1 / 2}$ as $\xi \rightarrow \infty$. Let $H(\eta ; Z, K)$ be the solution of Mathieu's equation on $-\mathbf{n} \leqq \eta \leqq n$ such that $H(0 ; Z, K)=h^{(1)}(0 ; Z, K)$ and $d H(0 ; Z, K) / d \eta$ $=i d h^{(1)}(0 ; Z, K) / d \xi$. Then $F=d H(\mathbf{n} ; Z, K) / d \eta$.

The horizontal line $C$ lies below all of the $Z_{n}(K)$ as $K \rightarrow \infty$.

We show that for every positive integer $N$ the contour integral is equal to the sum of the residues $R_{n}\left(\xi, \eta ; \xi_{0}, \eta_{0}: K\right), n=1,2, \cdots, N$, of $I$ at the poles $Z_{n}(K)$, and a remainder $\int_{C^{\prime}} I_{N}\left(\xi, \eta ; \xi_{0}, \eta_{0}: Z, K\right) d Z$. Here $C^{\prime}$ is the contour in the 4th quadrant of the $Z$-plane consisting of (i) the line $\operatorname{Im} Z=\operatorname{Im} Z_{N+1}$ from $\operatorname{Re} Z=-\infty$ to $\operatorname{Re} Z$ $=\operatorname{Re} Z_{N+1}-\left|Z_{N+1}-Z_{N}\right| / 2$, (ii) the lower half of the circle $\left|Z-Z_{N+1}\right|$ $=\left|Z_{N+1}-Z_{N}\right| / 2$, (iii) the line $\operatorname{Im} Z=\operatorname{Im} Z_{N+1}$ from $\operatorname{Re} Z=\operatorname{Re} Z_{N+1}$ $+\left|Z_{N+1}-Z_{N}\right| / 2$ to $\operatorname{Re} Z=-\epsilon, \epsilon>0$, (iv) the line $\operatorname{Re} Z=-\epsilon$ from $\operatorname{Im} Z=\operatorname{Im} Z_{N+1}$ to $\operatorname{Im} Z=+\infty$.

We prove that $\int_{C^{\prime}} I_{N} d Z=O\left(R_{N+1}\left(\xi, \eta ; \xi_{0}, \eta_{0}: K\right)\right)$ as $K \rightarrow \infty$, uniformly in $\xi$ and $\eta$, for all $(\xi, \eta)$ such that $(x, y) \in S_{<}\left(x_{0}, y_{0}\right)$.

Finally, we establish that if $(x, y) \in S_{<}\left(x_{0}, y_{0}\right)-C$, or if $(x, y)$ $\in S_{<}\left(x_{0}, y_{0}\right) \cap C$ the leading term of the asymptotic expansion of $R_{n}$ is the $n$th diffraction mode of the geometrical theory of diffraction.

In conclusion we remark that if $(x, y)$ and $\left(x_{0}, y_{0}\right)$ both lie on $C$ the series $\sum_{1}^{\infty} R_{n}$ converges to $G$. Otherwise, this series does not represent $G$, in fact it does not converge. This is in contrast to scattering by an ellipse (see [3] and [4]), a sphere (see [5]), and a parabola (see [6]). For in each of these cases there is a series representation of the solution that is also an asymptotic expansion in the shadow. 


\section{REFERENCES}

1. J. B. Keller, Diffraction by a convex cylinder, Trans. I.R.E. Ap-4 (1956), 312-321.

2. C. O. Bloom, Diffraction by a hyperbola, University Microfilms 26(1965), \#65-6793, 384.

3. F. Ursell, Creeping modes in a shadow, (to appear).

4. B. R. Levy, Diffraction by an elliptic cylinder, J. Math. Mech. (2)9(1960), 147-166.

5. H. M. Nussenzweig, High frequency scattering by an impenetrable sphere, Ann. of Phys. 34(1965), 23-95.

6. J. B. Keller and B. R. Levy, Decay exponents and diffraction coefficients for surface waves on surfaces of nonconstant curvature, I.R.E. Trans. Ap-7 (1959), Special Supplement.

Courant Institute of Mathematical Sciences, New York University 